\title{
Vorwort
}

Die gute Positionierung des neuen HGB-Kommentars im Markt und die gesetzlichen Entwicklungen erlauben uns, nach drei Jahren die zweite Auflage des Bilanzrecht-Kommentars aus der „Blauen Reihe“ des Otto Schmidt Verlags, den HKMS, vorzulegen. Das gewählte Konzept wird beibehalten, da wir die handelsrechtlichen Bilanzierungsregeln bewusst um die gesellschafts- und steuerrechtlich relevanten Bilanzierungsfragen ergänzen, um so eine Einheit zu erreichen und die inneren Verbindungen der Rechtsgebiete aufzuzeigen. Neu aufgenommen sind der Anhang zum Maßgeblichkeitsgrundsatz und die Kommentierung der $\$ \$ 341 \mathrm{q}-341 \mathrm{y}$ HGB (Konzernzahlungsbericht). Zudem wird nunmehr das gesamte Publizitätsgesetz (PublG) kommentiert. Das Werk wurde insgesamt überarbeitet und aktualisiert. Insbesondere die Regelungen aus ARUG II (Gesetz zur Umsetzung der zweiten Aktionärsrechterichtlinie) sowie der neu gefasste Deutsche Corporate Governance Kodex (DCGK) wurden umfassend eingearbeitet. Auf die zu erwartenden Neuerungen aus ESEF (Entwurf eines Gesetzes zur weiteren Umsetzung der Transparenzrichtlinie-Änderungsrichtlinie im Hinblick auf ein einheitliches elektronisches Format für Jahresfinanzberichte) und der COVID-19-Gesetzgebung wird hingewiesen. Neue Bilanzrechtsprechung des BFH, des BGH und des EuGH, die überarbeiteten Deutschen Rechnungslegungs Standards sind ebenso wie neue Verlautbarungen des Berufsstands der Wirtschaftsprüfer berücksichtigt. Der Rechtsstand bezieht sich auf den 31. Mai 2020.

Ohne das Engagement unseres Autorenteams wäre es nicht möglich gewesen, das Werk zu diesem Zeitpunkt vorlegen zu können. Dafür bedanken wir uns ausdrücklich. Aber nicht nur bei den Autorinnen und Autoren der Kommentierung möchten wir uns bedanken, auch unseren Mitarbeiterinnen und Mitarbeitern gilt unser Dank. Dank gilt auch dem Verlag und seinen Mitarbeitern, insbes. Frau RAin Eva Maria Marzinkowski, die uns beharrlich motiviert und unterstützt hat. Herausgeber und Verlag freuen sich, die zweite Auflage des HGB-Bilanzrecht-Kommentars - des HKMS - in die Hände der Nutzer zu legen. Inhaltliche Anregungen senden Sie bitte wieder unmittelbar an die Herausgeber (bilanzrecht@uni-hohenheim.de).

Stuttgart/Wien im Juli 2020

Die Herausgeber

\section{Vorwort der 1. Auflage}

Rechnungslegung, Jahresabschlussprüfung und Offenlegung sind Rechtsgebiete, die durch die EU, aber auch durch die internationale Rechnungslegung in ständiger Bewegung sind. Allein in den letzten Jahren gab es zwei EU-Richtlinien zur Rechnungslegung, die einen umfassenden Einfluss auf die Rechnungslegung nach HGB hatten. Durch diese Entwicklung dürfte aber auch deutlich werden, dass das HGB weiter eine wichtige Rolle bei der Gestaltung des Bilanzrechts hat und haben wird und nicht durch die IFRS abgelöst wird. Nach der Finanz- und Wirtschaftskrise ist auch die Sicht auf eine vorsichtsgeprägte, umsatzorientierte, verlustantizipierende Gewinn- und Vermögensermittlung, die mit dem HGB erreicht wird, neu bewertet worden. Die Bedeutung der IFRS jenseits der börsennotierten Unternehmen ist nach der massiven Kritik am HGB in den 1990er Jahren mittlerweile einer realistischen Einordnung gewichen. Weitgehend zeitgleich wurden die EUVerordnung zur Abschlussprüfung von Unternehmen von öffentlichem Interesse und die geänderte $\mathrm{Ab}$ schlussprüferrichtlinie veröffentlicht. Soweit diese Regelungen nicht direkt in Deutschland anzuwenden sind, wurden mittlerweile alle Regelungen vom deutschen Gesetzgeber in nationales Recht transformiert.

Diese ungebrochene Relevanz des HGB als Teil der steuerlichen und gesellschaftsrechtlichen Regelungen hat uns auch bewogen, im Otto Schmidt Verlag einen neuen HGB-Kommentar zum Bilanzrecht einschließlich der für die Rechnungslegung relevanten Normen der Steuerbilanz, der Abschlussprüfung, der Offenlegung und des Gesellschaftsrechts herauszugeben. Die Bezüge des Bilanzrechts zum Steuer- und Gesellschaftsrecht haben auch die inhaltlichen Ausführungen geprägt, ohne mit den jeweiligen themenspezifischen Kommentaren in Wettbewerb treten zu wollen. Neben den einschlägigen Paragraphen des HGB zur Rechnungslegung ( $\$ 238 \mathrm{ff}$. HGB), Abschlussprüfung ( $\$ 316 \mathrm{ff}$. HGB) und Offenlegung ( $\$ 325 \mathrm{ff}$. HGB) werden auch die im HGB, GmbHG oder AktG noch spezifischen Regelungen kommentiert; dass die Vorschriften für Genossenschaften und Großunternehmen nach PublG betrachtet werden, ist selbstverständlich, die branchenspezifischen Regelungen für Kreditinstitute, Versicherungsunternehmen und für Unternehmen des Rohstoffsektors wurden ausgespart. 
Eine besondere Beachtung finden die Normen des Steuerbilanzrechts und die hierzu ergangene Rechtsprechung des BFH. Aufgrund des Grundsatzes der Maßgeblichkeit ist die BFH-Rechtsprechung für das Handelsbilanzrecht nach wie vor von enormer Bedeutung; darüber hinaus wird auch die Rechtsprechung des EuGH zum Bilanzrecht in den einschlägigen Paragraphen beachtet. Die Regelungen des DRSC werden vor dem Hintergrund gewürdigt, dass für diese Regelungen vermutet wird, bei Bekanntmachung durch das Bundesministerium der Justiz den Charakter von Grundsätzen ordnungsmäßiger Buchführung der (Konzern-) Rechnungslegung zu haben. Von den Bereichen abgesehen, in denen die IFRS einen unmittelbaren Einfluss auf die Normen des HGB gefunden haben, werden die Vorschriften bewusst nicht kommentiert. Unsere Erfahrung hat gezeigt, dass vergleichende Betrachtungen mittlerweile obsolet sind. Wer konkrete Antworten auf Fragen der IFRS-Rechnungslegung sucht, wird in den einschlägigen - auch internationalen - Werken fündig und ist an den konkreten Regelungen, weniger einer Gegenüberstellung mit den HGB-Regeln interessiert. Im Rahmen der Kommentierung der Paragraphen zur Abschlussprüfung wurden die Prüfungsstandards und Prüfungshinweise des Instituts der Wirtschaftsprüfer in Deutschland e.V. (IDW) kritisch gewürdigt. Auch wenn diese Vorschriften nicht verbindlich sind, stellen sie eine wichtige Entscheidungshilfe für den Anwender dar.

Nach unserer Einschätzung kann das deutsche Bilanzrecht nicht mehr ohne diese Verbindungen zum Steuerund Gesellschaftsrecht betrachtet werden. Der vorliegende Kommentar zum Bilanzrecht sieht sich daher auch als Angelpunkt zu den hervorragenden und gut platzierten Kommentierungen des Otto Schmidt Verlags zum Steuer- und Gesellschaftsrecht. Diese Scharnierfunktion ist und war uns ein Anspruch und Ansporn. Die Positionierung und Ausrichtung spiegelt sich auch in unserer Zusammensetzung als Herausgeberteam, das nicht nur durch jeweils zwei Ökonomen und Juristen gebildet wird, sondern in dem nicht nur universitäre Lehre und Forschung, sondern auch die anwaltliche Kompetenz und Berufserfahrung eines Wirtschaftsprüfers vertreten ist. Diese Vielfalt findet sich auch bei den Verfassern der Kommentierung zu den einzelnen Paragraphen. Wir sind als Herausgeber stolz darauf, dass wir renommierte Persönlichkeiten aus Wissenschaft und Praxis, Juristen und Ökonomen als Autorinnen und Autoren für das vorliegende Werk gewinnen konnten. Basierend auf einem Vertrauensverhältnis zwischen Herausgebern und Autorinnen und Autoren spiegelt sich die daraus resultierende unproblematische Abstimmung der einzelnen Kommentierungen in der unseres Erachtens hohen Qualität der Kommentierung. Ohne das Engagement unseres Autorenteams wäre es nicht möglich gewesen, das Werk zu diesem Zeitpunkt vorlegen zu können. Dafür möchten wir uns ausdrücklich bedanken. Die Ausführungen berücksichtigen den Stand der Gesetzgebung, Rechtsprechung und Literatur bis Ende Juli 2017.

Der hier vorgelegte Bilanzrecht-Kommentar wendet sich nicht nur an die Angehörigen der wirtschaftsprüfenden und rechtsberatenden Berufe sowie Bilanzierende, sondern auch an Richterschaft und Kolleginnen, Kollegen und Studierende an Universitäten und andere Hochschulen. Um für die anwaltliche und anwenderorientierte Tagespraxis schnelle Informationen zu liefern, haben wir auf eine ausführliche Diskussion wissenschaftlicher Theorien verzichtet, wenngleich diese beim Aufbau der einzelnen Kommentierungen ihren Niederschlag gefunden haben.

Wir als Herausgeber bedanken uns aber nicht nur bei den Autorinnen und Autoren der Kommentierung, sondern auch bei unseren Mitarbeiterinnen und Mitarbeitern, ohne die ein solches Werk nicht entstehen kann. Dank gilt auch dem Verleger und seinen Mitarbeitern, die uns bei der Konzeption ebenso wie bei vielen Einzelfragen in nimmermüder Weise wirksam unterstützt haben. Herausgeber und Verlag freuen sich, einen neukonzipierten HGB-Bilanzrechtskommentar in die Hände der Nutzer zu legen. Inhaltliche Anregungen werden unmittelbar an die Herausgeber erbeten (bilanzrecht@uni-hohenheim.de). 